\title{
Multi-scale analysis of turbulence in the Earth's current sheet
}

\author{
M. Volwerk ${ }^{1,3}$, Z. Vörös ${ }^{1}$, W. Baumjohann ${ }^{1}$, R. Nakamura ${ }^{1}$, A. Runov ${ }^{1}$, T. L. Zhang ${ }^{1}$, K.-H. Glassmeier ${ }^{2}$, \\ R. A. Treumann ${ }^{3}$, B. Klecker ${ }^{3}$, A. Balogh ${ }^{4}$, and H. Rème ${ }^{5}$ \\ ${ }^{1}$ IWF, ÖAW, Graz, Austria \\ ${ }^{2}$ TU Braunschweig, Germany \\ ${ }^{3}$ MPE Garching, Germany \\ ${ }^{4}$ Imperial College, London, UK \\ ${ }^{5}$ CESR, Toulouse, France
}

Received: 26 August 2003 - Revised: 4 May 2004 - Accepted: 14 May 2004 - Published: 14 July 2004

Part of Special Issue "Spatio-temporal analysis and multipoint measurements in space"

\begin{abstract}
A multi-scale analysis of magnetotail turbulence in the Earth's tail current sheet is presented based on Cluster magnetometer observations. Both Fourier and wavelet analysis is used to describe the spectral index and scaling indices of the turbulence for different frequency regions. Flows in the tail are very important for driving the observed turbulence. There is a strong correlation between the maximum perpendicular flow velocity and the turbulence power for maximum velocities $150 \leq v_{\perp, \max } \leq 400 \mathrm{~km} / \mathrm{s}$. At higher maximum flow velocities the turbulence power levels out, showing a saturation of the generation mechanism. The suspected presence of breaks in the slope of the spectrum at two frequencies $\left(f_{1}\right.$ and $f_{2}$ ) can be confirmed for $f_{1} \approx 0.08 \mathrm{~Hz}$, but based on the data analysis the second break at $f_{2}$ is expected at a frequency higher than $12.5 \mathrm{~Hz}$, where the data cannot significantly be evaluated. A schematic turbulence power spectrum is presented based on the Cluster magnetic field measurements. Dependent on the presence of BBFs the spectral index or scaling index varies significantly.
\end{abstract}

Key words. Magnetospheric physics (magnetotail; plasma sheet; plasma waves and instabilities)

\section{Introduction}

The frequency scalings of the spectral power in the Earth's magnetotail current sheet have been studied by many (see, e.g. Hoshino et al., 1994; Borovsky et al., 1997; Zelenyi et al., 1998; Sigsbee et al., 2001; Borovsky and Funsten, 2003; Volwerk et al., 2003; Vörös et al., 2003). The goal of such investigations is to (1) come to a general view of the spectral properties of the turbulence; (2) what spectral indices to expect for which frequency intervals; (3) where and when to expect a break in the spectral index; (4) the di-

Correspondence to: M. Volwerk

(martin.volwerk@assoc.oeaw.ac.at) mensionality of the turbulence and (5) an indication of the power cascade. This paper is mainly concerning points 1,2 and 3; for points 4 and 5 we would like to direct the reader to Volwerk et al. (2003), Vörös et al. (submitted, 2004¹) and Borovsky and Funsten (2003) and references therein. Many schematics have been presented in which it is posited that there are three different spectral indices: $p_{1}$ for $f<f_{1} ; p_{2}$ for $f_{1}<f<f_{2}$ and $p_{3}$ for $f>f_{2}$. For example, Hoshino et al. (1994) have shown that $f_{1} \approx 0.04 \mathrm{~Hz}$ in Geotail data taken at $x_{\mathrm{GSM}} \approx 205 R_{\mathrm{E}}$, whereas Volwerk et al. (2004) find $f_{1} \approx 0.08 \mathrm{~Hz}$ from Cluster data taken at $R \approx 19 R_{\mathrm{E}}$.

In this paper we combine and expand on the results from Volwerk et al. (2003) and Vörös et al. (2003). In these papers the data from the magnetic field experiment (Balogh et al., 2001) are investigated for their turbulence scaling properties, combined with flow information from the plasma experiment (Rème et al., 2001). The plasma flows are probably the main drivers of the turbulence in the current sheet and, therefore, they play an important role in our study. Discussions on the characteristics of high-speed plasma flows, or bursty bulk flows (BBFs), can be found in, for example, Baumjohann et al. (1990), Angelopoulos et al. (1994) and Nakamura et al. (2002). We will show that the turbulent spectra have a break at low frequencies, $f_{1}$, but our data are insufficient to show a break at high frequencies, $f_{2}$. We also show that the break at $f_{1}$ is strongly related to BBFs, and that at high frequencies the spectral index shows large differences between flow and no-flow intervals.

The data that are used in this paper were obtained when the Cluster spacecraft had their apogee in the Earth's magnetotail at a radial distance of $\sim 19 R_{E}$. The inter-spacecraft separation distance was $1800 \mathrm{~km}$ in 2001 and $4000 \mathrm{~km}$ in 2002 . Using the full capabilities of the Cluster magnetometer up to a

\footnotetext{
${ }^{1}$ Vörös, Z., Baumjohann, W., Nakamura, R., Volwerk, M., Runov, A., Zhang, T. L., Eichelberger, H. U., Treumann, R., Georgescu, E., Balogh, A., Klecker, B., and Rème, H.: Magnetic turbulence in the plasma sheet, J. Geophys. Res., submitted, 2004.
} 

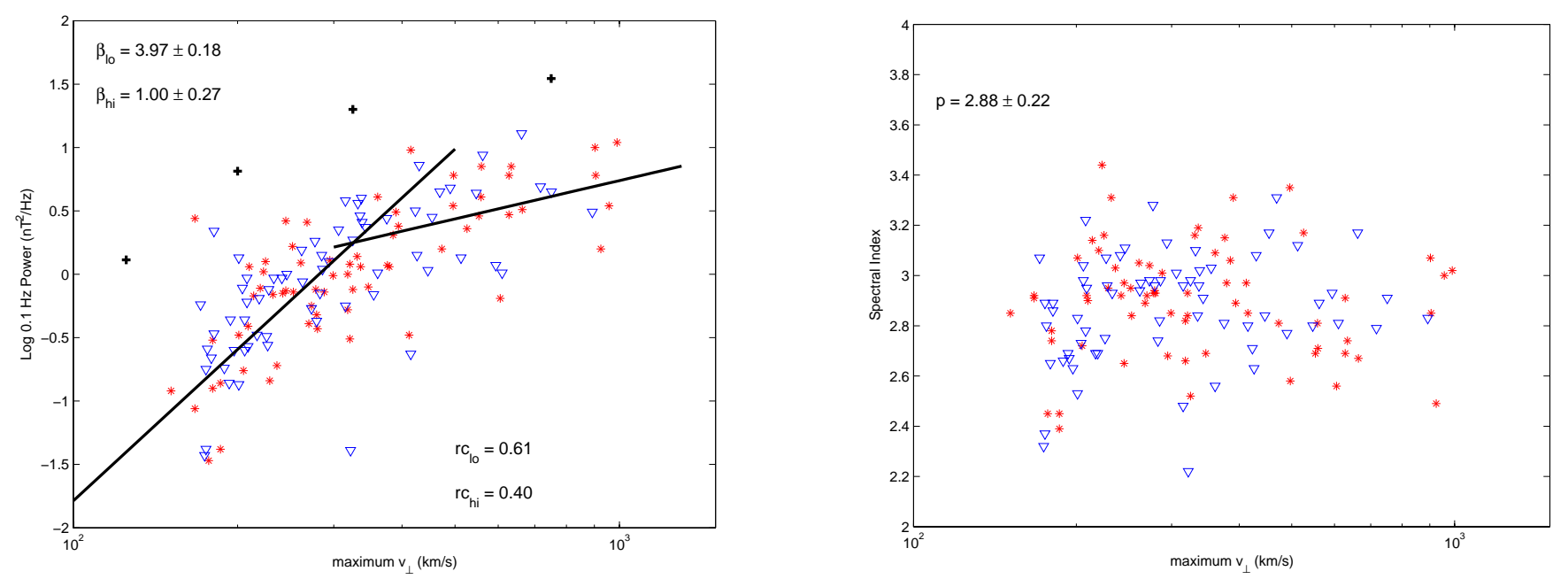

Fig. 1. The top panel shows the low frequency turbulence for $0.08 \leq f \leq 1 \mathrm{~Hz}$ for 68 intervals of 12 min near the neutral sheet. The left panel shows the total power in the turbulence as a function of the maximum perpendicular flow velocity during the 12-min interval. The stars and triangles represent $\mathrm{C} 1$ and $\mathrm{C} 3$, respectively. The pluses show the velocity dependence of the spectral power determined by Bauer et al. (1995a) for the frequency interval $0.03 \leq f \leq 0.13 \mathrm{~Hz}$. The velocity dependence of the spectral power is fitted for velocity intervals $150 \leq v_{\perp, \max } \leq 400 \mathrm{~km} / \mathrm{s}$ and $350 \leq v_{\perp, \max } \leq 1100 \mathrm{~km} / \mathrm{s}$. The bottom panel shows the spectral index as a function of maximum perpendicular flow velocity.

sampling rate of $67 \mathrm{~Hz}$, we will construct a spectral diagram for a broad frequency range, taking into account various dependencies on, i.e. the flow velocity of the plasma in the tail.

\section{Data analysis techniques}

We will first review the data analysis techniques that have been used in the papers that form the basis of this paper.

\subsection{High-velocity low-frequency spectral analysis}

An analysis of the low-frequency turbulence in the Earth's current sheet has been performed by Volwerk et al. (2003). They used 12-min intervals of data near the neutral sheet, covering the local time region between 21:00 and 03:00 LT, for both 2001 and 2002. The data were transformed to a mean field-aligned coordinate system. The spectral analysis was performed on $2 \mathrm{~Hz}$ sampled data, giving a Nyquist frequency of $f_{\mathrm{Ny}}=1 \mathrm{~Hz}$ and the spectra were averaged over 7 harmonics with a frequency resolution of $\Delta f=1.4 \mathrm{mHz}$. It was found that the power in the compressional component was much stronger than the left- and right-hand polarised wave power (see also Bauer et al., 1995a; 1995b; Volwerk et al., 2004). A lower limit was set to the maximum perpendicular flow velocity, $v_{\perp, \max }>150 \mathrm{~km} / \mathrm{s}$. Only then does the frequency interval in which we are interested, $0.08 \leq f \leq 1 \mathrm{~Hz}$, show power above the magnetometer noise level.

A summary plot of all data is shown in Fig. 1. For the turbulent power in the frequency range $0.08 \leq f \leq 1.0 \mathrm{~Hz}$ they found that it was highly dependent on the maximum perpendicular flow velocity, $v_{\perp \text {,max }}$ measured during the interval.
The spectral index of the turbulence is shown to be $p=2.88 \pm 0.22$ with no dependence on the flow velocity. This value is much larger than Kolmogorov $(p=5 / 3)$ and close to what one would expect for quasi-2-D turbulence. For pure 2-D turbulence one would expect that $p=3$ (Frisch, 1995). The occurrence of quasi-2-D turbulence is dependent on a region that is limited in one direction on scales longer than the extension of the region in the third dimension. This is natural for the Earth's current sheet, which is limited in the $z$-direction.

Interestingly it is found that the power in the turbulence can be described as:

$P \equiv P(f, v) \propto f^{-p} v^{\beta}$,

so not only is there the usual frequency dependence of the spectral power, $f^{-p}$, but also a velocity dependence $v^{\beta}$. In the paper by Volwerk et al. (2003) the data were split up in three local time regions: pre-midnight (12:00-23:00 LT); midnight (23:00-01:00 LT) and postmidnight (01:00-03:00 LT). It was shown that two different regions could be identified for the velocity dependence. For $v_{\perp, \max } \leq 400 \mathrm{~km} / \mathrm{s}$ the turbulent power increases strongly with slopes $\beta_{\text {pre }}=4.51 \pm 0.24, \quad \beta_{\text {mid }}=3.82 \pm 0.65$ and $\beta_{\text {post }}=3.45 \pm 0.16$ (in the total data set we find $\beta_{\mathrm{lo}}=3.76 \pm 0.21$, see Fig. 1 ).

We transform the frequency spectra to wave number space under the assumption that the slope of the spectrum does not change (Borovsky et al., 1997), i.e. $P(f) \propto f^{-p} \rightarrow P(k) \propto k^{-p}$. This results in a wave spectrum:

$P \equiv P(k, v) \propto v^{\beta-p} k^{1-D-p}$, 
where $D$ is the dimensionality of the problem, in this case $D=1$ (see Volwerk et al., 2003 for a discussion of this value). This dependence on the flow velocity can be explained when the wave power is generated by a streaming instability, most likely the Kelvin-Helmholtz instability (KHI) on the boundary between the flow channel and the ambient magnetotail. Transformed to wave number space one finds that the power grows linearly with the maximum velocity $\left(\beta_{\mathrm{lo}} \approx 3.76\right.$, $p \approx 2.88$ ), what one would expect for the KHI:

$\dot{P}=2 \gamma P$ with $\gamma \propto v$.

At higher velocities, $v_{\perp, \max }>400 \mathrm{~km} / \mathrm{s}$, the spectral power becomes saturated, demonstrated by a much smaller slope for the velocity dependence. For this region it was found that $\beta_{\text {pre }}=1.95 \pm 0.20, \beta_{\text {mid }}=1.20 \pm 0.45$ and $\beta_{\text {post }}=1.44 \pm 0.50$ (in the total data set we find $\beta_{\mathrm{hi}}=1.06 \pm 0.48$, see Fig. 1). One finds that the spectral power becomes independent from the flow velocity or even inversely dependent on it $\left(\beta_{\mathrm{hi}} \approx 1.06\right.$, $p \approx 2.88$ ), showing clearly the saturation of the generating instability. Indeed, Melrose (1986) states that "if the KHI develops too fast, the resuling turbulent mixing can reduce the shear below this threshold, thereby suppressing the instability".

For even lower frequencies $f \leq 0.06 \mathrm{~Hz}$, containing the $\mathrm{Pi} 2$ band $(8-25 \mathrm{mHz})$, the 12 -min intervals are too short to obtain an accurate spectral index. We will return to this frequency band later in the case studies in Sect. 3 .

\subsection{High-frequency wavelet analysis}

In order to investigate higher-frequency turbulence than in the previous section, we cannot suffice with $2-\mathrm{Hz}$ sampled data and just regular spectral analysis. The signals at higher frequencies are embedded in a high noise region. Therefore, for selected events, Vörös et al. (2003; submitted, 2004 ${ }^{1}$ ) studied the normal and burst mode $(22 \mathrm{~Hz}$ and $67 \mathrm{~Hz}$, respectively) magnetic field data with a discrete wavelet technique. Again, a relationship is sought between the spectral power and the frequency:

$P(f) \propto c_{f} f^{-\alpha_{\mathrm{S}}}$,

where now $c_{f}$ and $\alpha_{\mathrm{S}}$ are determined by a wavelet estimator. This estimator involves a semi-parametric wavelet technique based on a fast pyramidal algorithm, which allows unbiased estimations of the scaling parameters $c_{f}$ and $\alpha$ (Abry et al., 2000). A discrete wavelet transform of the time series is performed over a dyadic grid of scale $(j)$ and time $(\mathrm{t})$ and for each octave the variance $\mu_{j}$ of the discrete wavelet coefficients is calculated:

$\mu_{j}=\frac{1}{n_{j}} \sum_{t=1}^{n_{j}} d^{2}(j, t) \propto 2^{j \alpha} c_{f}$,

where $n_{j}$ are the number of coefficients at octave (scale) $j$ and $d(j, t)$ is the discrete wavelet coefficient. The parameters $c_{f}$ and $\alpha$ are then found by linear regression of the logarithm of the variance $\mu$ with respect to the observed scale $j$

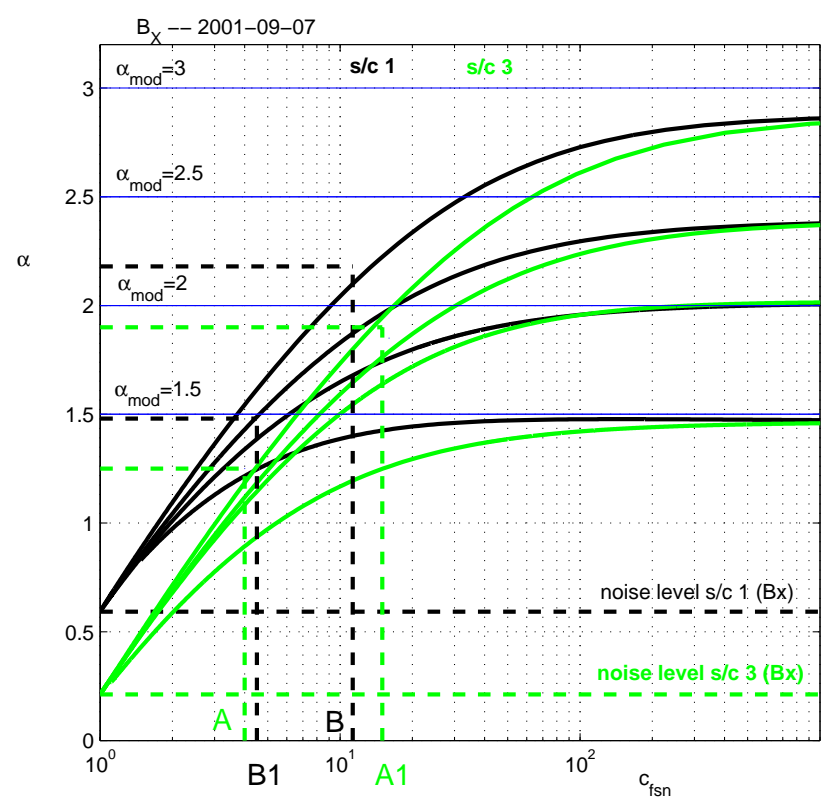

Fig. 2. The scaling parameter correction curves for two spacecraft (C1 and C3) for scale $j=4$. For known signal-to-noise ratios, $c_{\mathrm{fsn}}$, it is shown from synthetic data that for small values the true scaling $\alpha$ is recovered incorrectly and needs to be corrected. The graph also shows the difference in noise level for $\mathrm{C} 1$ and $\mathrm{C} 3$. A, B, A1 and B1 represent the examples studied in the case studies, respectively, for 27 August 2001 and 13 September 2002.

(intercept and slope, respectively); for a full description, see Vörös et al. (2004). We choose to describe the "spectral index" by $\alpha_{S}$, to clarify the different analysis technique used.

As at higher frequencies the signal-to-noise ratio becomes very small; a check of the feasibility of this procedure is needed. Therefore, Vörös et al. (submitted, 2004 ${ }^{1}$ ) have used quiet magnetotail intervals and embedded artificial signals, with known scaling parameters and reminiscent of signals expected to be found in the magnetotail, and performed the wavelet analysis to find these scaling parameters. It was shown that depending on the signal-to-noise ratio, $c_{\mathrm{fsn}}$ and on the scale $j$, recovery of the scaling parameters was possible. For different frequency ranges (or scales $j$ ) the time window used was optimized to recover the synthetic signals. By varying the signal-to-noise level, the known scaling paremeters were recovered with varying accuracy, and were usually underestimated. The correction curves showed the correct value of $\alpha_{\mathrm{S}}$ for a measured $\alpha_{\mathrm{S}, \mathrm{m}}$. Covering a large parameter space of $c_{\mathrm{fsn}}$ and $j$, Vörös et al. (submitted, 2004 ${ }^{1}$ ) created these correction curves which can be applied to the determination of the scaling parameters from active magnetotail data. The correction curves were computed by embedding artificial signals to quiet time measurements on 7 September 2001. Examples of these curves for $\mathrm{C} 1$ and $\mathrm{C} 3$ can be seen in Fig. 2, and these curves will be used in this paper.

The main problems in this process are: determining the signal-to-noise ratio, $c_{\text {fsn }}$ (the noise level for each spacecraft is different); and the width of the window in which the 


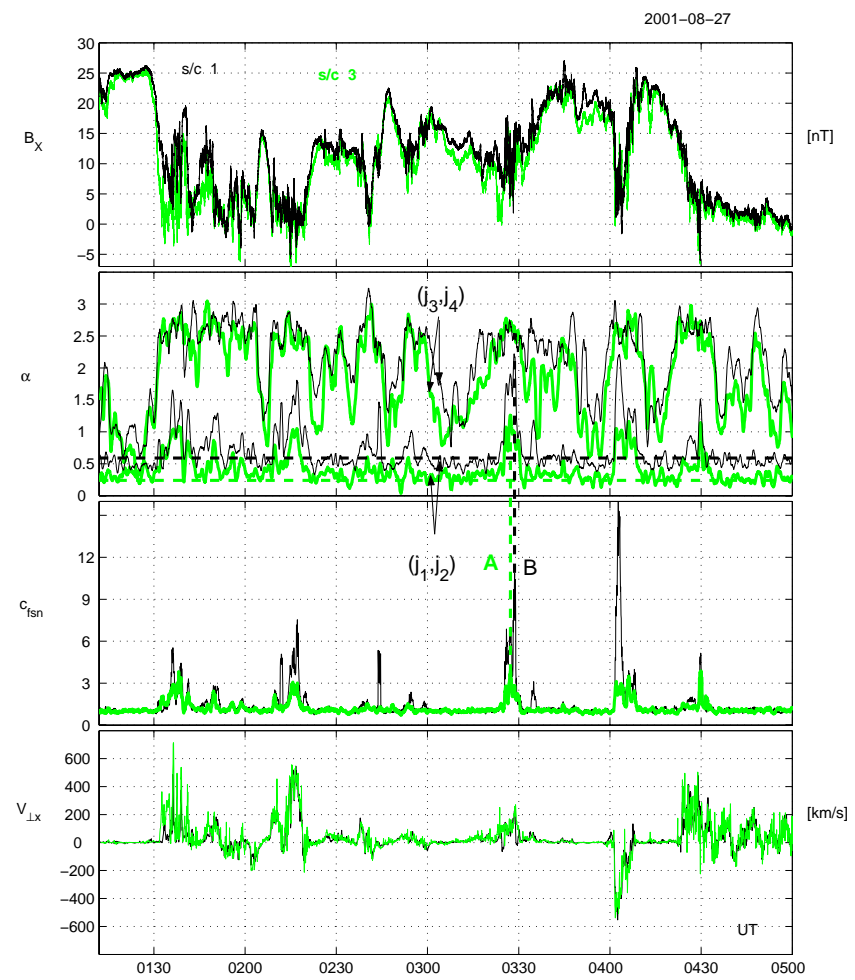

Fig. 3. Top panel: The $B_{\mathrm{X}}$ for 27 August 2001, for $\mathrm{C} 1$ (black) and C3 (green). Next panel: The wavelet scaling parameter $\alpha$ for scales $\left(j_{1}, j_{2}\right)$ and $\left(j_{3}, j_{4}\right)$. Next panel: The signal-to-noise estimator $c_{\mathrm{fsn}}$ defined as the relative power of intercepts $\left.c_{\mathrm{fsn}}=c_{\text {activity }} /<c_{\text {noise }}\right\rangle$. Bottom panel: $x$-component of the perpendicular flow velocity. The correction of the scaling parameter $\alpha_{\mathrm{S}}$ for $\mathrm{A}$ and $\mathrm{B}$ are shown in Fig. 2.

wavelet transform is performed. The latter problem can be solved by using a synthetic data set and for each scale the optimal width of the window can be found by trial. The different noise levels for each spacecraft can be determined. This makes sure that each spacecraft has its own set of correction curves, as shown in Fig. 2. The first problem is not so trivial, but using the data one can construct a quantity determined by the "relative power of intercepts" from the wavelet analysis. In the wavelet scalogram the intercept at scale $j=0$ is determined for the noise (from the quiet time data) and for active data, i.e. the $c_{\mathrm{f}} \mathrm{s}$ describing the spectral power $P(f)$. From analysis of synthetic data one finds that this relative power of intercepts, $c_{\mathrm{fsn}}=c_{\text {activity }} /\left\langle c_{\text {noise }}\right\rangle$, is an adequate quantity to describe the signal-to-noise ratio. We note that $\left\langle c_{\text {noise }}\right\rangle$ is independent of the location of the Cluster spacecraft; for further details, see Vörös et al. (submitted, 2004 ${ }^{1}$ ).

For selected intervals containing bursty bulk flows (BBFs; see, e.g. Baumjohann et al., 1990; Angelopoulos et al., 1994) the (corrected) scaling parameters have been determined. It is found that in the absence of flow in the magnetotail $c_{\mathrm{fsn}} \approx 1$, and only during fast flows does one find that $c_{\mathrm{fsn}}>1$ and is scaling information available. From the correction curves in Fig. 2 it is clear that for $c_{\mathrm{fsn}} \ll 100$ the scaling parameter $\alpha$ for small scales $\left(j_{1}, j_{2}\right)=(0.08,0.33)$ s needs to be corrected,
Table 1. Statistical evaluation of large-scale $\alpha_{\mathrm{LS}}$ for 27 August 2001, for two different conditions. Cond. 1: Based on small scales: BBF when $\alpha_{\mathrm{SS}}>0.9$ and $c_{\mathrm{fsn}}>2$; non-BBF when $\alpha_{\mathrm{SS}}<0.4$ and $c_{\mathrm{fsn}}<1.1$; Cond. 2 : Based on perpendicular velocity: BBF when $v_{\perp, \mathrm{xy}}>300 \mathrm{~km} / \mathrm{s} ;$ non-BBF when $v_{\perp, \mathrm{xy}}<100 \mathrm{~km} / \mathrm{s}$.

\begin{tabular}{llccc} 
& & $B_{\mathrm{X}}$ & $B_{\mathrm{y}}$ & $B_{\mathrm{Z}}$ \\
& & $\mathrm{C} 1$ & $\mathrm{C} 1$ & $\mathrm{C} 1$ \\
\hline Cond. & $\alpha_{\mathrm{LS}}$ BBF & $2.55 \pm 0.04$ & $2.56 \pm 0.06$ & $2.53 \pm 0.08$ \\
1 & $\alpha_{\mathrm{LS}}$ non-BBF & $1.7 \pm 0.3$ & $2.1 \pm 0.3$ & $2.1 \pm 0.2$ \\
\hline Cond. & $\alpha_{\mathrm{LS}}$ BBF & $2.6 \pm 0.1$ & $2.6 \pm 0.1$ & $2.6 \pm 0.1$ \\
2 & $\alpha_{\text {LS }}$ non-BBF & $1.9 \pm 0.3$ & $2.1 \pm 0.3$ & $2.1 \pm 0.3$ \\
\hline & & $\mathrm{C} 3$ & $\mathrm{C} 3$ & $\mathrm{C} 3$ \\
\hline Cond. & $\alpha_{\mathrm{LS}}$ BBF & $2.59 \pm 0.04$ & $2.59 \pm 0.04$ & $2.52 \pm 0.06$ \\
1 & $\alpha_{\mathrm{LS}}$ non-BBF & $1.6 \pm 0.4$ & $1.8 \pm 0.4$ & $1.9 \pm 0.2$ \\
\hline Cond. & $\alpha_{\mathrm{LS}}$ BBF & $2.6 \pm 0.1$ & $2.6 \pm 0.1$ & $2.5 \pm 0.1$ \\
2 & $\alpha_{\mathrm{LS}}$ non-BBF & $1.5 \pm 0.4$ & $1.7 \pm 0.4$ & $2.0 \pm 0.3$ \\
\hline
\end{tabular}

whereas at larger scales $\left(j_{3}, j_{4}\right)=(0.7,11)$ s the scaling parameters can be trusted. The $c_{\mathrm{fsn}}$ seems to correlate best with the perpendicular plasma flow, $v_{\perp}$.

Figure 3 shows the wavelet analysis results for $B_{\mathrm{x}}$ for 27 August 2001. During the interval 01:10-05:00 UT on 27 August 2001, the Cluster spacecraft were near the $z_{\mathrm{GSM}}=0$ plane, in the post-midnight magnetotail ( $\left.x_{\mathrm{GSM}} \sim-19 R_{E}\right)$. The correlation between $v_{\perp}$ and $c_{\text {fsn }}$ is clear, whenever there are BBFs the signal-to-noise ratio increases. This is similar to what was observed in the low-frequency turbulence, where the perpendicular flow velocity had to exceed $150 \mathrm{~km} / \mathrm{s}$ for the whole frequency interval to show power above the noise level. The results from the wavelet analysis can be found in Table 1. Two different kinds of conditions are put onto the data:

1. Based on small scales: BBF when $\alpha_{\mathrm{SS}}>0.9$ and $c_{\mathrm{fsn}}>2$; non-BBF when $\alpha_{\mathrm{SS}}<0.4$ and $c_{\mathrm{fsn}}<1.1$;

2. Based on perpendicular velocity: $\mathrm{BBF}$ when $v_{\perp, \mathrm{xy}}>300 \mathrm{~km} / \mathrm{s}$; non-BBF when $v_{\perp, \mathrm{xy}}<100 \mathrm{~km} / \mathrm{s}$.

For both conditions on the data we find that for BBF type of data the scaling $\alpha_{\mathrm{LS}} \approx 2.6$, where the large scale indicates time scales of $0.7-11 \mathrm{~s}$ or frequency scale of $1.4-0.09 \mathrm{~Hz}$, overlapping with the spectral analysis region in the previous section. Indeed, we find that during fast flows the scaling index is similar between the two methods (i.e. spectral analysis and wavelet analysis), $2.88 \pm 0.22$ and $2.6 \pm 0.1$. In the case of non-BBF periods we find that $1.5 \leq \alpha_{\mathrm{LS}} \leq 2.1$, which is very close to a Kolmogorov spectrum $\left(\alpha_{\mathrm{kol}}=5 / 3\right)$.

For the smaller scales, i.e. $\left(j_{1}, j_{2}\right)=(0.08,0.33) \mathrm{s}$, or for the frequency range $12.5-3 \mathrm{~Hz}$ it was found that the $c_{\text {fsn }} \ll 100$ (see Fig. 3); thus the values for $\alpha_{\mathrm{SS}}$ must be corrected using the curves in Fig. 2. This shows that for the identified regions of BBFs the wave power can be described 

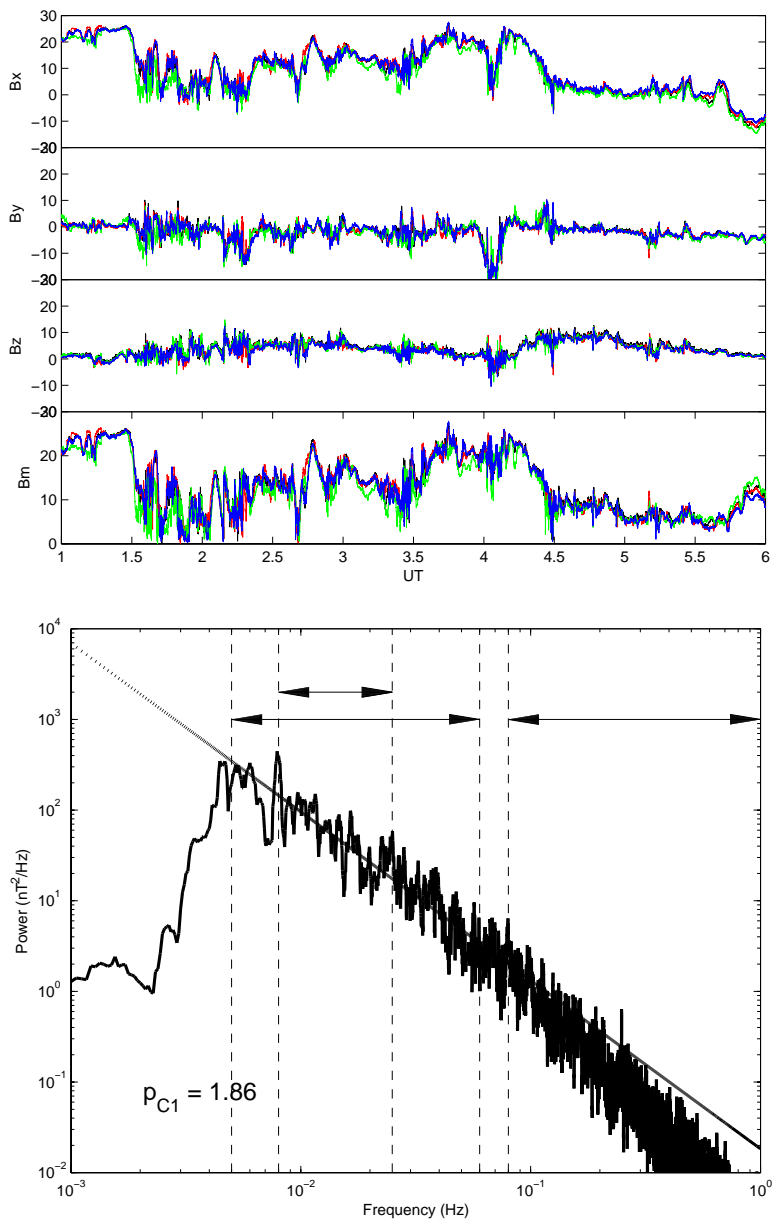

Fig. 4. Top panel: magnetic field data for 27 August 2001, in GSM coordinates for all spacecraft, colour coded in the following way: $\mathrm{C} 1$ black, $\mathrm{C} 2$ red, $\mathrm{C} 3$ green and $\mathrm{C} 4$ blue. Bottom panel: The power spectra of the compressional components of the magnetic field for $\mathrm{C} 1$. The spectral power is fitted with respect to frequency over the interval $0.005 \leq f \leq 0.04 \mathrm{~Hz}$ to obtain a low-frequency spectral index and shown as the solid straight line. The arrows depict the different frequency ranges: the low frequency fit range (long arrow left), the $\mathrm{Pi} 2$ frequency band (short arrow left) and the high-frequency range (long arrow right).

by $\alpha_{\mathrm{SS}} \approx 2.6$, similar to what was found for the larger scale $\left(j_{3}, j_{4}\right)$. In the case of no BBF activity the wavelet analysis shows that $c_{\mathrm{fsn}} \approx 1$, which means that only noise is measured and thus, we can conclude that there is no significant energy flow to small scales.

\section{Case studies}

We will study in more detail two extended periods with the Cluster spacecraft near the neutral sheet, during substorm times, and with the availability of burst mode $(67 \mathrm{~Hz})$ magnetic field data. The days are: 27 August 2001, 01:00-06:00 UT (burst mode data for 01:30-03:00 UT) and 13 September 2002, 17:00-23:00 UT (burst mode data for
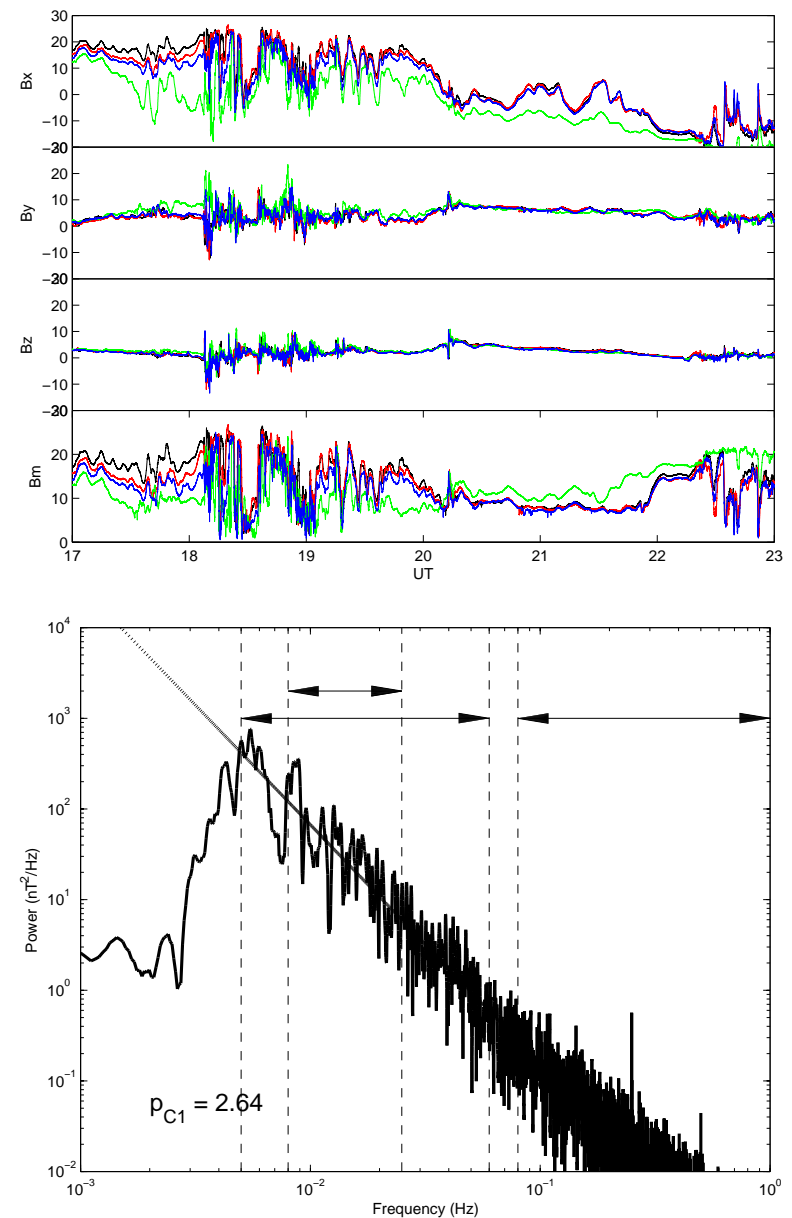

Fig. 5. Magnetic field data and power spectrum for 13 September 2002, for details, see caption Fig. 4.

18:00-20:00 UT). Some of the results for the first interval have already been discussed in the previous section.

The magnetic field data for 27 August are given in Fig. 4. With a long time interval there is enough spectral resolution to fit the low-frequency region of the spectrum. We choose a frequency interval $0.005 \leq f \leq 0.06 \mathrm{~Hz}$. We find that we can describe the spectrum with a spectral index $p=1.8$. This is flatter than the higher frequency interval $0.08<f<1.0 \mathrm{~Hz}$, which is described by a spectral index of $p=2.8$.

For the spectral power at higher frequencies we need the wavelet analysis approach. In the previous section we found that the description of the spectral power was dependent on the presence of BBF activity. For BBF intervals we find that for $0.09 \leq f \leq 1.4 \mathrm{~Hz}$ the wavelet scaling is described by $\alpha_{\mathrm{LS}} \approx 2.6$ and for $3.0 \leq f \leq 12 \mathrm{~Hz}$ the scaling is described by $\alpha_{\mathrm{SS}} \approx 2.6$ as well, indicating that there is no break in the power spectrum between these two frequency ranges. On the other hand, for the non-BBF intervals it was found that $1.5 \leq \alpha_{\mathrm{LS}} \leq 2.1$ and for higher frequencies the signal-to-noise ratio was too small to obtain a result.

The magnetic field data for 13 September 2002 are given in Fig. 5. Using spectral analysis we find that for the 

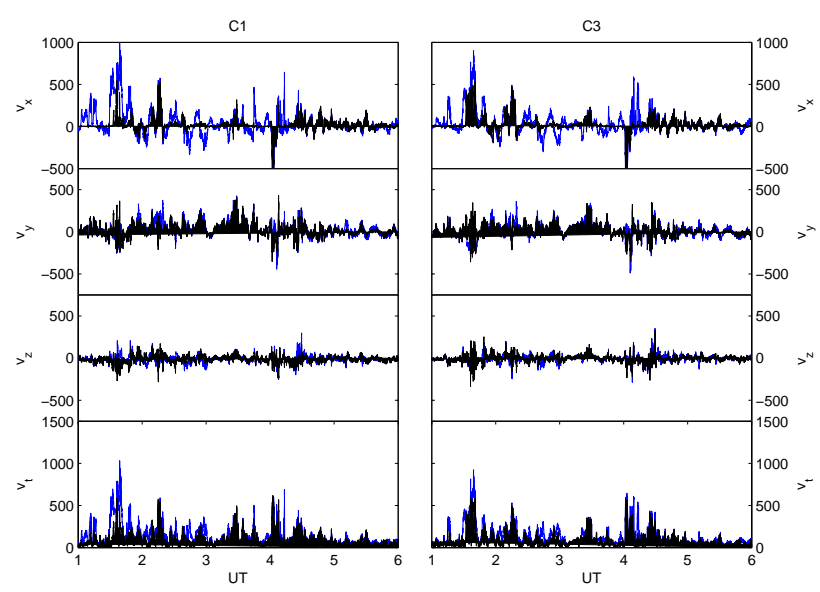

Fig. 6. The plasma flow velocity for 27 August 2001, with the perpendicular component shown as the filled curve underneath, for $\mathrm{C} 1$ and $\mathrm{C} 3$.
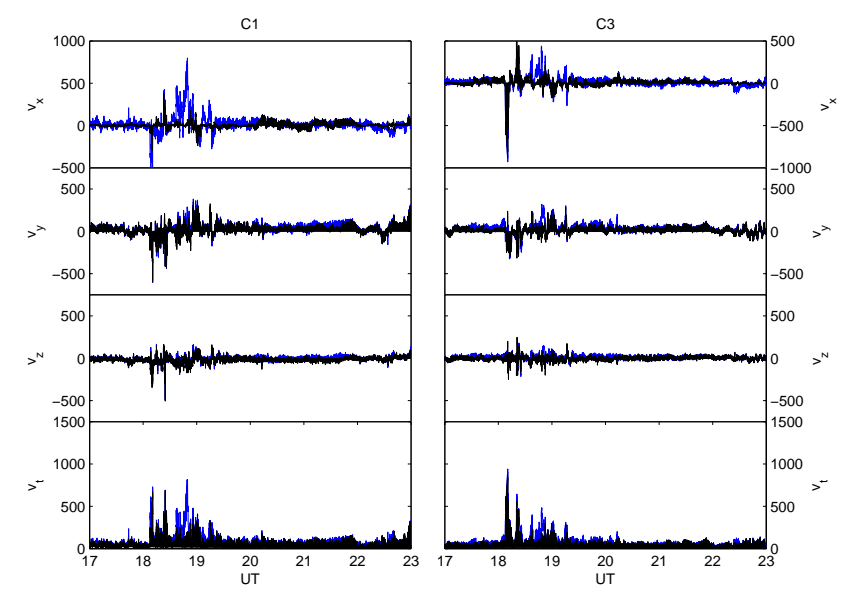

Fig. 7. The plasma flow velocity for 13 September 2002, with the perpendicular component shown as the filled curve underneath, for $\mathrm{C} 1$ and $\mathrm{C} 3$.

low-frequency region of the spectrum the wave power can be described by $p \approx 2.6$, and the higher frequency range has a similar slope, indicating that there is no break in the spectrum. This is a notable difference from the slope for $27 \mathrm{Au}-$ gust 2001, where such a break was found and which is also described in Hoshino et al. (1994) and Volwerk et al. (2004). The break in the power spectrum near $f \approx 0.08 \mathrm{~Hz}$ was explained by the finite thickness of the fast flow channel. Volwerk et al. (2003) assumed a thickness of $4000 \mathrm{~km}$ for the flow channel and found that magneto-acoustic waves would have an "eigenmode" at a frequency $f \approx 0.06 \mathrm{~Hz}$.

A clear difference between 27 August 2001 and 13 September 2002 can be seen in Figs. 6 and 7. The latter day has a much shorter interval of strong flow activity. Indeed, when we perform spectral analysis on the data from 13 September 2002, for the interval 19:00-23:00 UT, i.e. take out the strong flow bursts, the spectral index remains approximately the same. Only the total spectral power decreases
Table 2. Statistical evaluation of large-scale $\alpha_{\mathrm{LS}}$ for 13 September, 2002, for two different conditions. Cond. 1: Based on small scales: BBF when $\alpha_{\mathrm{SS}}>0.9$ and $c_{\mathrm{fsn}}>2$; non-BBF when $\alpha_{\mathrm{SS}}<0.4$ and $c_{\mathrm{fsn}}<1.1$; Cond. 2 : Based on perpendicular velocity: $\mathrm{BBF}$ when $v_{\perp, \mathrm{xy}}>300 \mathrm{~km} / \mathrm{s}$; non-BBF when $v_{\perp, \mathrm{xy}}<100 \mathrm{~km} / \mathrm{s}$.

\begin{tabular}{llccc} 
& & $B_{\mathrm{x}}$ & $B_{\mathrm{y}}$ & $B_{\mathrm{Z}}$ \\
& & $\mathrm{C} 1$ & $\mathrm{C} 1$ & $\mathrm{C} 1$ \\
\hline Cond. & $\alpha_{\mathrm{LS}}$ BBF & $2.3 \pm 0.2$ & $2.2 \pm 0.2$ & $2.4 \pm 0.3$ \\
1 & $\alpha_{\text {LS non-BBF }}$ & $1.0 \pm 0.3$ & $1.2 \pm 0.3$ & $2.0 \pm 0.1$ \\
\hline Cond. & $\alpha_{\text {LS BBF }}$ & $2.42 \pm 0.15$ & $2.5 \pm 0.2$ & $2.4 \pm 0.2$ \\
2 & $\alpha_{\text {LS non-BBF }}$ & $1.3 \pm 0.5$ & $1.7 \pm 0.5$ & $2.2 \pm 0.2$ \\
\hline & & $\mathrm{C} 3$ & $\mathrm{C} 3$ & $\mathrm{C} 3$ \\
\hline Cond. & $\alpha_{\text {LS BBF }}$ & $2.5 \pm 0.2$ & $2.3 \pm 0.2$ & $2.3 \pm 0.3$ \\
1 & $\alpha_{\text {LS non-BBF }}$ & $0.9 \pm 0.4$ & $1.0 \pm 0.5$ & $1.3 \pm 0.5$ \\
\hline Cond. & $\alpha_{\text {LS BBF }}$ & $2.5 \pm 0.1$ & $2.4 \pm 0.2$ & $2.6 \pm 0.1$ \\
2 & $\alpha_{\text {LS non-BBF }}$ & $1.4 \pm 0.6$ & $1.5 \pm 0.6$ & $1.6 \pm 0.6$ \\
\hline
\end{tabular}

by a factor $\sim 2$. Apparently, the presence of a flow channel is necessary for the creation of a break in the power spectrum. Without limited $z$-dimensions the magneto-acoustic waves can propagate over the whole current sheet, creating a coupling between small and large scales.

The results of the wavelet analysis of 13 September 2002 are shown in Fig. 8 and the statistical evaluation of the largescale $\alpha_{\mathrm{LS}}$ for this event are listed in Table 2. The observed interval in burst mode is shorter than for the previous case ( $\sim 2 \mathrm{~h}$ vs. $\sim 4 \mathrm{~h}$ ). It is clear from Fig. 8 that there are only a few intervals with flow activity. This indicates that the statistics are not as good as in the previous case.

Based on the same condition criteria as above, the data are split up into BBF and non-BBF events. Again, it is shown that $\mathrm{BBF}$ events after correction show scaling parameters $\alpha_{\mathrm{LS}} \approx 2$.4. For non-BBF events the determination of $\alpha_{\mathrm{LS}}$ becomes difficult, as $c_{\mathrm{fsn}}$ becomes very small and the correction curves in Fig. 2 are very close together.

\section{Multi-scalar turbulent spectrum}

Combining all the results from above we can come to a full description of the turbulent wave spectrum. Naturally we have to take into account the different conditions of the current sheet to obtain such a description. From our results above the spectral index can be very different depending on the flow activity. We list the spectral index $p$ and scaling parameter $\alpha_{\mathrm{S}}$ in Table 3 .

Using the values in Table 3 we can obtain a multiscale spectrum of the turbulence in the current sheet. Indeed, we can now put values to the posited spectrum by Zelenyi et al. (1998) and expand on the power spectrum presented by Borovsky and Funsten (2003) (but note that Borovsky and Funsten investigate totally different magnetospheric conditions, magnetotail compression by northward IMF magnetic 
Table 3. The spectral indices $p$ and scaling parameters $\alpha_{\mathrm{S}}$ as determined from the spectral and wavelet analysis. Average values for flow and no-flow intervals are given.

\begin{tabular}{|c|c|c|c|c|}
\hline $\begin{array}{c}\text { Freq. Range } \\
\mathrm{Hz}\end{array}$ & $\begin{array}{c}\text { flow } \\
p\end{array}$ & $\begin{array}{c}\text { no-flow } \\
p\end{array}$ & $\begin{array}{c}\text { flow } \\
\alpha_{\mathrm{S}}\end{array}$ & $\begin{array}{c}\text { no-flow } \\
\alpha_{\mathrm{S}}\end{array}$ \\
\hline $0.005 \leq f \leq 0.04$ & $\sim 1.8$ & $\sim 2.8$ & NA & NA \\
\hline $0.08 \leq f \leq 1.0$ & $\sim 2.8$ & NA & $\sim 2.6$ & NA \\
\hline $3.0 \leq f \leq 12.5$ & NA & NA & $\sim 2.6$ & $\sim 1.8$ \\
\hline
\end{tabular}

clouds, whereas we investigate substorm-related phenomena). We find that there is one possible spectral break at $f_{1} \approx 0.08 \mathrm{~Hz}$, and no real evidence for a second spectral break at higher frequencies. The smaller scaling parameter $\alpha_{\mathrm{LS}}$ for the high frequency range must be regarded critically, as the signal-to-noise ratios for non-BBF events are very small and thus, some of the information in the data may be lost in the noise. In Fig. 9 we show the results as obtained in this paper. The solid boxes show the slopes for BBF conditions and the striped boxes for non-BBF conditions. The shaded regions show the possible variation in slopes as given by the error bars.

\section{Discussion}

We have investigated the spectral power scaling with respect to frequency for the magnetic wave activity observed in the Earth's tail current sheet. It was proposed that in the power spectra there would be two breaks at frequencies usually labelled $f_{1}$ and $f_{2}$ or $f_{*}$ and $f_{* *}$. It was shown by Hoshino et al. (1994) that $f_{1} \approx 0.04 \mathrm{~Hz}$ and similarly by Volwerk et al. (2004) that $f_{1} \approx 0.08 \mathrm{~Hz}$ and Bauer et al. (1995a) noticed a break in the spectrum $f_{1} \approx 0.03 \mathrm{~Hz}$. This seems to be a well established frequency range for $f_{1}$. However, Zelenyi et al. (1998) studied the $z$-component of the magnetic field and found that it showed a "two-kink behaviour" with turn-over frequencies $f_{*} \approx 0.01 \mathrm{~Hz}$ and $f_{* *} \approx 0.25 \mathrm{~Hz}$. This is not in agreement with the results presented in this paper. It is not feasible to put $f_{1} \equiv f_{*}$. Also, our results show no indication of a break at $f_{* *} \approx 0.25 \mathrm{~Hz}$.

An interesting detail in our results is that in the frequency range $0.08 \leq f \leq 1 \mathrm{~Hz}$, which was investigated both by Fourier spectral analysis and wavelet analysis, the spectral index was 2.6 and 2.8 , respectively. In the intermediate frequency range there is strong overlap between the powers determined from spectra and wavelet analyses under flow conditions, suggesting that the spectral index is more of the order of 2.6 \pm 0.1 . Physically this implies that the turbulence is not strictly twodimensional in the presence of strong flows, but rather it is intermittent such that the dimensionality is fractal $2<D<3$ with scales substantially smaller than the width of the region (in this case the flow channel) in $z$-direction contributing. The presence of smaller scale structures contributing to the turbulence is also suggested by the index in the high-

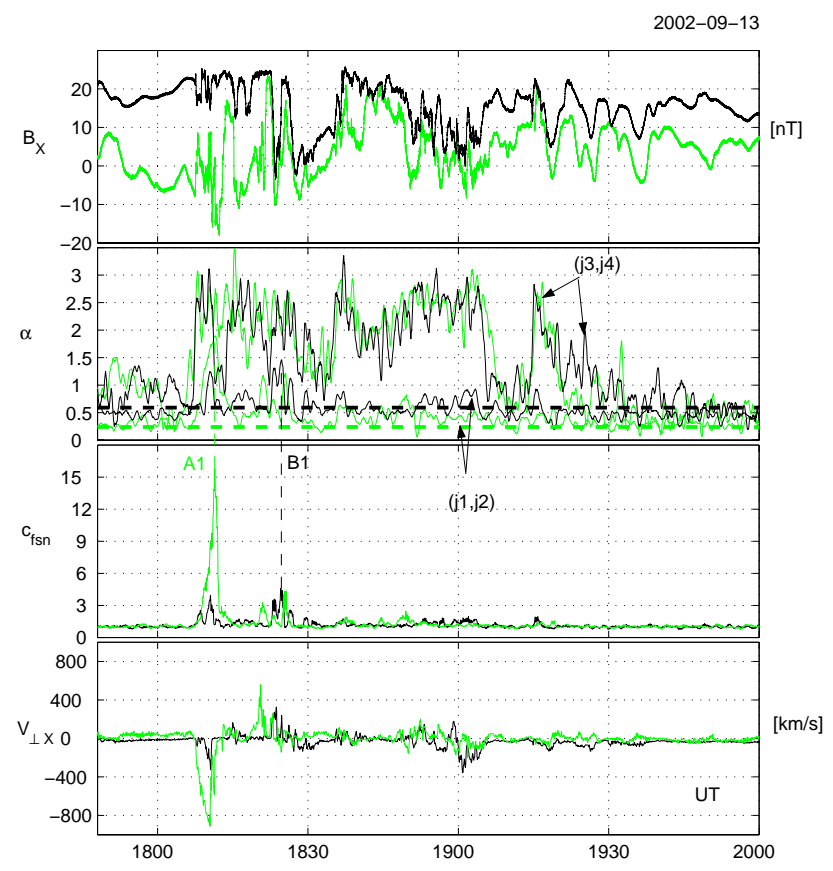

Fig. 8. Wavelet analysis of the data from 13 September 2002 in similar form as Fig. 3. The correction of the scaling parameter $\alpha_{\mathrm{S}}$ for A1 and B1 are shown in Fig. 2.

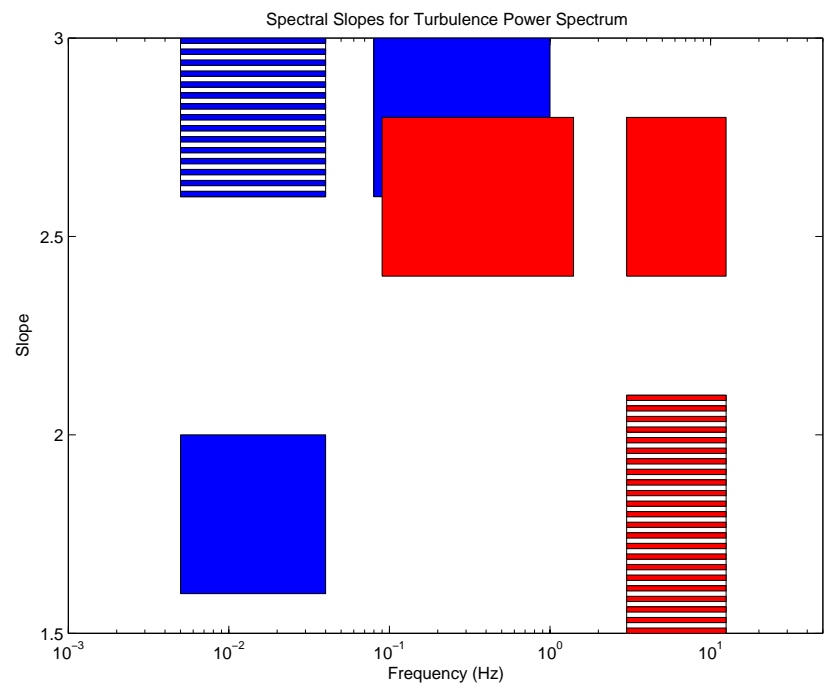

Fig. 9. A schematic for the slope of the power spectrum as deduced in this paper for different frequency ranges, determination methods and flow activity. Blue: Spectral analysis; Red: Wavelet analysis. The solid boxes are for BBF intervals and the striped boxes are for non-BBF periods. For the numerical values of the slopes, see Table 3 .

frequency range found from the wavelet analysis, which is identical to that in the intermediate frequency range, suggesting no spectral break at those higher frequencies.

It is interesting to also comment on the flat Kolmogorovlike spectra obtained from the spectral analysis in the very low-frequency range. This quasi-three-dimensional 
character of the turbulence here suggests that scales considerably larger than the width of the region under consideration are involved at those frequencies. The local properties and in particular the boundedness of the region in the $z$-direction does not substantially affect the turbulence here. The strong spectral break at $f \approx 0.08 \mathrm{~Hz}$, where the spectrum changes from the flat Kolmogorov-like spectrum to the steep quasi-two-dimensional intermittent fractal turbulent spectrum, shows the transition from scales which ignore those that recognize the effect of the boundedness of the region in $z$.

The spectral behaviour under no-flow conditions shows the transition from quasi-two-dimensional turbulence at low frequencies to Kolmogorov-like turbulence at high frequencies. Since under these conditions the spectrum cannot be resolved by wavelet analysis, as mentioned earlier, the intermediate region index is lacking. However, if real, the break from steep, low-frequency 2-D turbulence to flat, high frequency 3-D turbulence indicates that the larger scales at low frequency are sensitive to the boundedness of the region. The smaller scales at high frequency are clearly independent of the presence of the boundaries of the region such that their behaviour is three-dimensional. The obvious conclusion that can be drawn from this mutual behaviour under no-flow and under flow conditions is that the turbulent region detected under no-flow conditions is wider in the $z$ direction than under flow conditions. Under high flow conditions the region of the plasma sheet that behaves turbulently must be thin compared to the conditions when no flows are detected. Nakamura et al. (2002) discusses the thinning of the current sheet during fast flows.

We note that the indices found in this paper are higher than previously estimated, where it was found that the spectral index is 2-2.5 (Bauer et al., 1995a), 2.2 (Borovsky et al., 1997) and 1.78-2.43 (Zelenyi et al., 1998, with no reference for the very low 1.78 given). This need not be surprising, as our data sets were highly selected with strong criteria on magnetic field strength and flow velocity. Volwerk et al. (2003) showed that if the flow velocity was too low $\left(v_{\perp, \max }<150 \mathrm{~km} / \mathrm{s}\right)$ the power in the higher-frequency part of the interval $0.08-1 \mathrm{~Hz}$ disappeared into the noise level of the magnetometer, and thus, artificially reduced the spectral index.

Unfortunately, we cannot give a value for $f_{2}$ at which the next break in the spectrum would occur. The possible break at $f \approx 3 \mathrm{~Hz}$ that can be seen in Fig. 9 cannot be interpreted as such. The change in scaling index $\alpha_{\mathrm{SS}}$ depends on the presence of BBFs. If there is no flow, then the signal-to-noise ratio is very small, and we lose information about the scaling index. The noise tends to flatten the slope of the spectrum, which is an artifact, and does not represent the true scaling index of the high-frequency turbulence. Therefore, we have to conclude that $f_{2}$ probably resides at a higher frequency than shown in the figure, in a region where we are unable to significantly investigate the data.

The periods of non-BBF show different characteristics. In the low frequency we see that the slope is steep ( $p \approx 2.8$ ), indicating that there is transport of turbulent power to larger scales, what one would expect for 2-D turbulence. In the high-frequency range, however, we see a flatter slope $(p \approx 1.8)$, indicating that there is no transport of turbulent power to these scales. The latter introduces an artificial break in the power spectrum, which is related to the signal disappearing into the noise level and not with a physical process as in the lower frequency range.

Acknowledgements. We would like to acknowledge the Cluster Science Data System (CSDS). The authors ould like to thank H.U. Eichelberger and Y. Bogdanova for preparing the Cluster FGM and CIS data. The work by MV and KHG was financially supported by the German Bundesministerium für Bildung und Forschung and the Zentrum für Luft- und Raumfahrt under contracts 50 OC 0104 and 50 OC 0103, respectively.

Topical Editor T. Pulkkinen thanks a referee for his help in evaluating this paper.

\section{References}

Abry, P., Flandrin, R., Taqqu, M. S., and Veitch, D.: Self-similar network traffic and performance, Wiley Interscience, New York, 2000.

Angelopoulos, V., Kennel, C. F., Coroniti, F. V., Pellat, R., Kivelson, M. G., Walker, R. J., Russell, C. T., Baumjohann, W., Feldman, W. C., and Gosling, J. T.: Statistical characteristics of bursty bulk flow events, J. Geophys. Res., 99, 21 257-21 280, 1994.

Balogh, A., Carr, C. M., Acuña, M. H., Dunlop, M. W., Beek, T. J., Brown, P., Fornacon, K.-H., Georgescu, E., Glassmeier, K.-H., Harris, J., Musmann, G., Oddy, T., and Schwingenschuh, K.: The Cluster magnetic field investigation: overview of in-flight performance and initial results, Ann. Geophys., 19, 1207-1217, 2001.

Bauer, T. M., Baumjohann, W., Treumann, R. A., Sckopke, N., and Lühr, H.: Low-frequency waves in the near-Earth plasma sheet, J. Geophys. Res., 100, 9605-9617, 1995a.

Bauer, T. M., Baumjohann, W., and Treumann, R. A.: Neutral sheet oscillations at substorm onset, J. Geophys. Res., 100, 23737 23 743, 1995b.

Baumjohann, W., Paschmann, G., and Lühr, H.: Characteristics of high-speed flows in the plasma sheet, J. Geophys. Res., 95, 3801-3809, 1990.

Borovsky, J. E. and Funsten, H. O.: MHD turbulence in the Earth's plasma sheet: Dynamics, dissipation, and driving, J. Geophys. Res., 108(A7), 1284, doi:1.1029/2002JA009625, 2003.

Borovsky, J. E., Elphic, R. C., Funsten, H. O., and Thomsen, M. F.: The Earth's plasma sheet as a laboratory for flow turbulence in high- $\beta$ MHD, J. Plasma Phys., 57, 1-34, 1997.

Frisch, U.: Turbulence, Cambridge University Press, Cambridge, UK, 1995.

Hoshino, M., Nishida, A., Yamamoto, T., and Kokubun, S.: Turbulent magnetic field in the distant magnetotail: Bottom-up process of plasmoid formation?, Geophys. Res. Lett., 21, 2935-2938, 1994.

Melrose, D. B.: Instabilities in space and laboratory plasmas, Cambridge University Press, 1986

Nakamura, R., Baumjohann, W., Runov, A., Volwerk, M., Zhang, T. L., Klecker, B., Bogdanova, Y., Roux, A., Balogh, A., Rème, H., Sauvaud, J. A., and Frey, H. U.: Fast flows dur- 
ing current sheet thinning, Geophys. Res. Lett., 29, (29), 2140, doi:10.1029/2002GL016200, 2002.

Rème, H., Aoustin, C., Bosqued, J., et al.: First multi-spacecraft ion measurements in and near the Earth's magnetosphere with the identical Cluster ion spectrometry (CIS) experiment, Ann. Geophys., 19, 1303-1354, 2001.

Sigsbee, K., Cattell, C. A., Moser, F. S., Tsuruda, K., and Kokubun, S.: Geotail observations of low-frequency waves from 0.001 to $16 \mathrm{~Hz}$ during the November 24, 1996, geospace environment modelling substorm challenge event, J. Geophys. Res., 106, 435445, 2001

Volwerk, M., Nakamura, R., Baumjohann, W., et al.: A statistical study of compressional waves in the tail current sheet, J. Geophys. Res., 108, (A12), 1429, doi:10.1029/2003JA101155, 2003.

Volwerk, M., Baumjohann, W., Glassmeier, K.-H., Nakamura, R., Zhang, T. L., Runov, A., Vörös, Z., Klecker, B., Treumann, R. A., Bogdanova, Y., Eichelberger, H.-U., Balogh, A., and Rème, H.: Compressional waves in the Earth's neutral sheet, Ann. Geophys., 22, 303-315, 2004.
Vörös, Z., Baumjohann, W., Nakamura, R., Runov, A., Zhang, T. L., Volwerk, M., Eichelberger, H. U., Balogh, A., Horbury, T. S., Glassmeier, K.-H., Klecker, B., and Rème, H.: Multi-scale magnetic field intermittence in the plasma sheet, Ann. Geophys., 21, 1955-1964, 2003.

Vörös, Z., Baumjohann, W., Nakamura, R., Runov, A., Volwerk, M., Zhang, T. L., and Balogh, A.: Wavelet analysis of magnetic turbulence in the Earth's plasma sheet, Phys. Plasmas, 11, 13331338, 2004.

Zelenyi, L., Milanov, A. V., and Zimbardo, G.: Multiscale magnetic structure of the distant tail: Self-consistent fractal approach, New perspectives on the earth's magnetotail, Geophys. Monogr. Ser., 105, edited by Nishida, A., Baker, D. N., and Cowley, S. W. H., AGU, Washington, DC, USA, 321-339, 1998. 